\title{
Electrodynamics in complex systems: Application to near-field probing of optical microresonators
}

\author{
Annick Castiaux \\ Institute for Studies in Interface Sciences, Facultés Universitaires Notre-Dame de la Paix, B-5000 Namur, Belgium \\ Christian Girard \\ Laboratoire de Physique Moléculaire, URA CNRS 772, Université de Franche Comté, F-25300 Besançon, France \\ Alain Dereux \\ Laboratoire de Physique et Optique Submicronique, URA CNRS 1796, Université de Bourgogne, F-21004 Dijon, France \\ Olivier J. F. Martin \\ Swiss Federal Institute of Technology, Eidgenössische Technische Hochschule Zentrum, CH-8092 Zurich, Switzerland \\ Jean-Pol Vigneron \\ Institute for Studies in Interface Sciences, Facultés Universitaires Notre-Dame de la Paix, B-5000 Namur, Belgium
}

(Received 7 March 1996)

\begin{abstract}
This paper discusses recent theoretical efforts to develop a general and flexible method for the calculation of the field distributions around and inside complex optical systems involving both dielectric and metallic materials. Starting from the usual light-matter coupling Hamiltonian, we derive a self-consistent equation for the optical field in arbitrary optical systems composed of $N$ different subdomains. We show that an appropriate solving procedure based on the real-space discretization of each subdomain raises the present approach to the rank of an accurate predictive numerical scheme. In order to illustrate its applicability, we use this formalism to address challenging problems related to nonradiative energy transfers in near-field optics. In particular, we investigate in detail the detuning of a microresonator probed by a near-field optical probe. [S1063-651X(96)01911-3]
\end{abstract}

PACS number(s): 42.25.-p, 61.16.Ch, 02.30.Tb, 02.60.Nm

\section{INTRODUCTION}

While the theory of the interaction of optical electromagnetic waves with macroscopic or microscopic objects is now well established, our theoretical knowledge on the scattering of light by mesoscopic systems (i.e., of size comparable to the wavelength) remains more limited. Since a growing number of experimental situations involve simultaneously mesoscopic and nanoscopic systems, incomplete information on the mesoscopic range negatively affects our understanding of the optical interaction at the nanoscale. In particular, the rapidly developing research on subwavelength surface optics (scanning near-field optical microscopy, ultrafast optoelectronic devices) calls for accurate theoretical frameworks able to account for nonradiative electromagnetic phenomena. A numerical method for solving Maxwell equations is needed because both geometries and dielectric responses of typical mesoscopic systems display a high degree of complexity. However, numerical methods traditionally used in electrodynamics are not well suited for the study of mesoscopic structures. Cumbersome procedures appear to be uncertain and cannot always produce reliable results. The main origin of these problems can be attributed to the crucial role played by the evanescent components of the field in the near-field zone close to mesoscopic scatterers. In complete analogy with the tunnel effect for electrons, these evanescent components can lead to optical tunnel effects. In the mesoscopic range, the accurate treatment of evanescent waves requires one to deal carefully with the electromagnetic boundary conditions at each interface and to include appropriate dielectric responses.

Recently, several variants of electromagnetic scattering theory based on Green's functions were applied successfully to the modelization of near-field optical phenomena [1-3]. Although Green's functions may be expanded in Fourier or multipoles series, most of these variants preferred a discretization in direct space. Indeed, experimentally relevant nearfield optical phenomena occur in highly spatially localized regions, which require a prohibitive number of Fourier components for an accurate treatment in Fourier space. Let us note that this Green's formalism has been used for a long time in quantum scattering problems. In electrodynamics, it has also been exploited extensively to solve engineering problems involving external sources of currents, where the solution in the source region was not required [4]. The application to the study of phenomena where the solution in the source region (such as in near-field optics) is of primary importance was hindered by the apparently divergent nature of the Green's dyadic in the source region. As discussed by Yaghjian, this divergence is related to depolarization effects and can be handled in a perfectly reliable manner for three-, two-, and one-dimensional systems [5].

The main difficulty in formulating a predictive theory aimed at studying scattering and light confinement effects generated around mesoscopic structures lies in the fact that any approximation cannot be reasonably applied. Actually, a faithful description of the field structure far away as well as in the immediate proximity of the particles needs the inclu- 
sion of both retardation effects and multiple reflections between each scatterer.

This paper outlines how the field susceptibility Green's function technique deals efficiently with these different points. More precisely, starting from a light-matter coupling Hamiltonian, we shall present in Sec. II a brief overview of the field susceptibility Green's function technique, which allows one to obtain general solutions of Maxwell's equations through a time-dependent vector Lippmann-Schwinger equation. This real-space approach is of course a fundamental advantage when dealing with nonperiodic arrangements composed of different mesoscopic structures of arbitrary shape and dielectric constant. In addition, we show that the dimensionality of the physical system under consideration leads to a specific set of equations able to reduce the discretization to domains associated with each interacting structure. From this flexible framework, we will address, in Sec. III, several challenging issues related to nonradiative energy transfer resulting from the near-field optical interaction between microresonators and dielectric probes similar to those used in near-field optics. In particular, we will investigate in detail the evolution of the complete optical field pattern as a function of the geometrical configuration. Finally, these results will be discussed in relation to recent experimental works.

\section{QUANTUM DERIVATION FOR SCATTERED OPTICAL FIELD IN COMPLEX SYSTEMS}

\section{A. Time-dependent Lippmann-Schwinger equation}

Let us consider a three-dimensional system of arbitrary shape and composition perturbed by an external optical field $\mathbf{E}_{0}(\mathbf{r}, t)$. The light-matter coupling Hamiltonian is given by [6]

$$
H(t)=-\int d \mathbf{r}\left[\mathbf{E}_{0}(\mathbf{r}, t)+\mathcal{E}(\mathbf{r}, t)\right] \cdot \mathcal{P}(\mathbf{r}, t),
$$

where $\mathcal{E}(\mathbf{r}, t)$ and $\mathcal{P}(\mathbf{r}, t)$ are, respectively, the field and polarization operators for the considered material system. In the interaction representation, these operators read

$$
\mathcal{E}(\mathbf{r}, t)=\exp \left[i H_{0} t / \hbar\right] \mathcal{E}(\mathbf{r}) \exp \left[-i H_{0} t / \hbar\right]
$$

and

$$
\mathcal{P}(\mathbf{r}, t)=\exp \left[i H_{0} t / \hbar\right] \mathcal{P}(\mathbf{r}) \exp \left[-i H_{0} t / \hbar\right]
$$

where $H_{0}$ is the Hamiltonian for the unperturbed system. Assuming that the wave functions of the different elements of the system are decorrelated, we are authorized to apply the time-dependent Hartree approximation, which supposes that each part of the system moves under the combined effect of the external force and the average displacements of the other parts of the system $[7,8]$. The linear responses corresponding to $\mathcal{E}(\mathbf{r}, t)$ and $\mathcal{P}(\mathbf{r}, t)$ are obtained from the perturbation theory

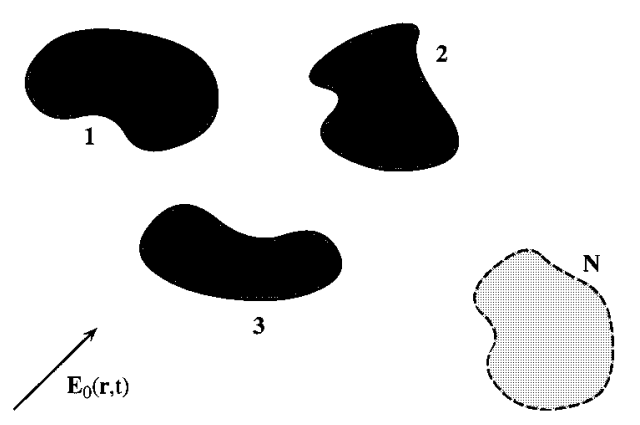

FIG. 1. Division of the considered 3D system into $N$ distinct subdomains.

$$
\begin{aligned}
\mathbf{E}(\mathbf{r}, t) & =\left\langle\mathbf{E}_{0}(\mathbf{r}, t)+\mathcal{E}(\mathbf{r}, t)\right\rangle \\
& =\mathbf{E}_{0}(\mathbf{r}, t)+\int_{-\infty}^{t} d t^{\prime} \int d \mathbf{r}^{\prime} \mathbf{S}\left(\mathbf{r}, \mathbf{r}^{\prime}, t-t^{\prime}\right) \cdot\left\langle\mathcal{P}\left(\mathbf{r}^{\prime}, t^{\prime}\right)\right\rangle
\end{aligned}
$$

and

$$
\begin{aligned}
\mathbf{P}(\mathbf{r}, t) & =\langle\mathcal{P}(\mathbf{r}, t)\rangle \\
& =\int_{-\infty}^{t} d t^{\prime} \int d \mathbf{r}^{\prime} \boldsymbol{\chi}\left(\mathbf{r}, \mathbf{r}^{\prime}, t-t^{\prime}\right) \cdot\left\langle\mathbf{E}_{0}\left(\mathbf{r}^{\prime}, t^{\prime}\right)+\mathcal{E}\left(\mathbf{r}^{\prime}, t^{\prime}\right)\right\rangle .
\end{aligned}
$$

In these coupled equations, the two dyadic tensors $\mathbf{S}\left(\mathbf{r}, \mathbf{r}^{\prime}, t-t^{\prime}\right)$ and $\boldsymbol{\chi}\left(\mathbf{r}, \mathbf{r}^{\prime}, t-t^{\prime}\right)$ represent, respectively, the field susceptibility of the reference medium and the nonlocal linear susceptibility of the considered three-dimensional (3D) system.

Introducing Eq. (5) into Eq. (4) gives the time-dependent Lippmann-Schwinger equation

$$
\begin{aligned}
\mathbf{E}(\mathbf{r}, t)= & \mathbf{E}_{0}(\mathbf{r}, t)+\int_{-\infty}^{t} d t^{\prime} \int_{-\infty}^{t^{\prime}} d t^{\prime \prime} \int d \mathbf{r}^{\prime} \\
& \times \int d \mathbf{r}^{\prime \prime} \mathbf{S}\left(\mathbf{r}, \mathbf{r}^{\prime}, t-t^{\prime}\right) \cdot \boldsymbol{\chi}\left(\mathbf{r}^{\prime}, \mathbf{r}^{\prime \prime}, t^{\prime}-t^{\prime \prime}\right) \cdot \mathbf{E}\left(\mathbf{r}^{\prime \prime}, t^{\prime \prime}\right) .
\end{aligned}
$$

Equation (6) can deal with any general time-resolved lightmatter problem. In order to be able to solve Eq. (6) we transform it into $\omega$ space by applying a Fourier transform on the time component. This leads to

$$
\begin{aligned}
\mathbf{E}(\mathbf{r}, \omega)= & \mathbf{E}_{0}(\mathbf{r}, \omega)+\int d \mathbf{r}^{\prime} \\
& \times \int d \mathbf{r}^{\prime \prime} \mathbf{S}\left(\mathbf{r}, \mathbf{r}^{\prime}, \omega\right) \cdot \chi\left(\mathbf{r}^{\prime}, \mathbf{r}^{\prime \prime}, \omega\right) \cdot \mathbf{E}\left(\mathbf{r}^{\prime \prime}, \omega\right)
\end{aligned}
$$

\section{B. Separation of the system into $N$ subdomains}

We now consider that our 3D system can be divided into $N$ distinct subdomains (see Fig. 1) having no interaction with each other in the absence of the external field $\mathbf{E}_{0}(\mathbf{r}, t)$. Each subdomain $D_{n}$ is characterized by a susceptibility 
$\boldsymbol{\chi}_{n}\left(\mathbf{r}, \mathbf{r}^{\prime}, \omega\right)$ that vanishes when $\mathbf{r}$ and $\mathbf{r}^{\prime}$ are not in the domain $D_{n}$. The total susceptibility of the entire system is then

$$
\boldsymbol{\chi}\left(\mathbf{r}, \mathbf{r}^{\prime}, \omega\right)=\sum_{n=1}^{N} \boldsymbol{\chi}_{n}\left(\mathbf{r}, \mathbf{r}^{\prime}, \omega\right)
$$

The Lippmann-Schwinger equation (7) can then be written as

$$
\begin{aligned}
\mathbf{E}(\mathbf{r}, \omega)= & \mathbf{E}_{0}(\mathbf{r}, \omega)+\sum_{n=1}^{N} \int d \mathbf{r}^{\prime} \int_{D_{n}} d \mathbf{r}^{\prime \prime} \mathbf{S}\left(\mathbf{r}, \mathbf{r}^{\prime}, \omega\right) \\
& \times \boldsymbol{\chi}_{n}\left(\mathbf{r}^{\prime}, \mathbf{r}^{\prime \prime}, \omega\right) \mathbf{E}\left(\mathbf{r}^{\prime \prime}, \omega\right)
\end{aligned}
$$

The second space integral in Eq. (9) is limited to the subdomain $D_{n}$ since the susceptibility $\boldsymbol{\chi}_{n}\left(\mathbf{r}, \mathbf{r}^{\prime}, \omega\right)$ vanishes when $\mathbf{r}^{\prime}$ does not belong to $D_{n}$. The contribution of each subdomain to the entire system is of two types. First, if $\mathbf{r}$ belongs to the considered subdomain, we obtain the self-interaction of $D_{n}$. Second, if $\mathbf{r}$ belongs to another subdomain, the contribution represents a coupling effect between these two domains. Considering successively each subdomain, we progressively modify the wave function of the entire system and finally obtain the total wave function corresponding to the entire system.

When the reference medium is vacuum, its field susceptibility $\mathbf{S}_{0}\left(\mathbf{r}, \mathbf{r}^{\prime}, \omega\right)$ is given by the well-known expression [9]

$$
\mathbf{S}_{0}\left(\mathbf{r}, \mathbf{r}^{\prime}, \omega\right)=\int d \mathbf{k}\left[\mathbf{1}-\frac{1}{k_{0}^{2}} \mathbf{k} \mathbf{k}\right] \frac{\exp [i \mathbf{k} \cdot \mathbf{R}]}{k_{0}^{2}-k^{2}}
$$

where $\mathbf{k}_{0}$ is the incident wave vector of the perturbating field and $\mathbf{R}=\left(\mathbf{r}-\mathbf{r}^{\prime}\right)$. In direct space, this field susceptibility reads [9]

$$
\mathbf{S}_{0}\left(\mathbf{r}, \mathbf{r}^{\prime}, \omega\right)=\left[\mathbf{1}-\frac{1}{k_{0}^{2}} \boldsymbol{\nabla} \boldsymbol{\nabla}^{\prime}\right] \frac{\exp [i \mathbf{k} \cdot \mathbf{R}]}{|\mathbf{R}|} .
$$

It is clear from the preceding expression that the field susceptibility presents a pole when $\mathbf{R}$ reaches zero, i.e., when the source point and the target point are the same. This singularity is removed by taking into account a depolarization term [5]. This will be shown in the Appendixes.

If we suppose that all the effects are purely local, the susceptibility $\boldsymbol{\chi}_{n}\left(\mathbf{r}, \mathbf{r}^{\prime}, \omega\right)$ for each subdomain reduces to $\boldsymbol{\chi}_{n}(\mathbf{r}, \omega)$. We can then write a simplified form of the Lippmann-Schwinger equation (9)

$$
\begin{aligned}
\mathbf{E}(\mathbf{r}, \omega)= & \mathbf{E}_{0}(\mathbf{r}, \omega) \\
& +\sum_{n=1}^{N} \int_{D_{n}} d \mathbf{r}^{\prime} \mathbf{S}_{0}\left(\mathbf{r}, \mathbf{r}^{\prime}, \omega\right) \boldsymbol{\chi}_{n}\left(\mathbf{r}^{\prime}, \omega\right) \mathbf{E}\left(\mathbf{r}^{\prime}, \omega\right)
\end{aligned}
$$

An adapted and independent discretization for each subdomain $D_{n}$ makes the resolution of this equation possible with high accuracy $[10,11]$.

In the application presented in Sec. III, the system under study holds a translational invariance. Equation (12) is particularized to such a two-dimensional configuration in Ap- pendix A. We will use this form of the field susceptibility in Sec. III to study a complex 2D system.

\section{AB INITIO STUDY OF A MICRORESONATOR}

\section{A. The context of near-field optics}

One of the main advantages of the formalism presented in the preceding section lies in the fact that it can handle complex inhomogeneous systems formed by distinct objects. Another important feature is its ability to investigate physical effects occurring at the close vicinity of these objects. Such effects, involving a high density of evanescent waves, play a dominant role in the new challenges brought up by near-field optics. Indeed, at the near-field level, most of the energy transfers between different objects are nonradiative transfers. These evanescent waves, first used as a tool to reach high resolution in optical microscopy [12-14], are now studied for themselves in extremely fundamental optical experiments.

Recently, several groups $[15,16]$ reported the possibility of mapping the optical field structure inside a Fabry-Pérot cavity by collecting with a pointed optical fiber the evanescent wave above the cavity. These experiments were studied in a theoretical work [17]. In the same context, whispering gallery modes occurring in high- $Q$ microspheres were also recently investigated using evanescent waves and nonradiative energy transfers between the sphere surface and a probing tip [18].

The microcavity presented in the next subsection is similar to a setup already used experimentally [15], but with a considerably reduced size. We will study the tuning of this small resonator and the effect of the geometry on its $Q$ factor. We will also consider the nonradiative transfer process taking place between this microcavity and a probing tip placed in its near vicinity. This problem presents the theoretical difficulties for which our approach is particularly well suited: first, the geometries of the involved objects are nontrivial and the material composition highly inhomogeneous; second, all the phenomena of interest are accessible by studying the behavior of evanescent waves and different nonradiative transfer processes.

\section{B. The 2D system under study}

The microresonator that we will investigate is a typical multidomain system as presented before. The resonator itself is composed by three distinct domains: a microprism, a mirror, and a semitransparent mirror (Fig. 2). A fourth element will be added in Sec. III C: a small probing tip above the prism surface. Two problems will be addressed in the following sections: the tuning of the resonator by moving the mirrors and the effect of a small probe in the near vicinity of the prism surface. The concept of subdomains is extremely interesting for investigating this complex system. In particular, it is evident that the freedom to move each subdomain independently is essential. Indeed, if we were constrained by a unique discretization grid for the entire system, the relative positioning of the different elements would be conditioned by this grid and we would not be able to easily study the two above-mentioned problems.

The description of our resonator is given in Fig. 2. A dielectric prism is inserted between two mirrors, one being 


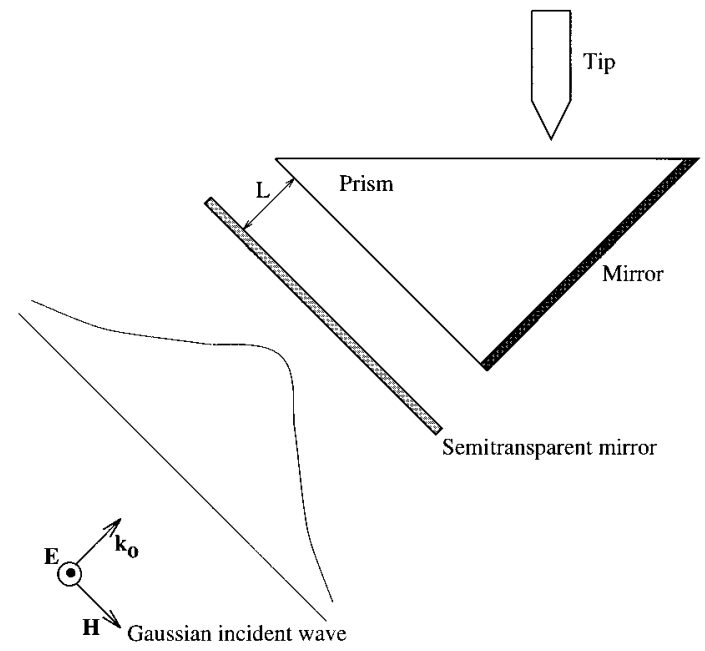

FIG. 2. Coupling of a microresonator with a small probe. The resonator is a dielectric prism inserted between two silver mirrors. A Gaussian $s$-polarized wave is incident on the prism in total internal reflection.

thin enough to let a small amount of incident light go through. The incident beam, with an $s$-polarized electric field and a wavelength in vacuum $\lambda=632 \mathrm{~nm}$, hits the prism hypotenuse at an angle larger than the critical angle, so that it produces total internal reflection in the prism. Multiple reflections on the mirrors and the three prism faces occur inside the cavity. If the configuration is favorable, we can obtain a high- $Q$ microresonator.

A good configuration was obtained with the following parameters. The prism has a dielectric constant of $\epsilon=3$. This value was chosen to enhance the total internal reflection and compensate the losses due to the small size of our system. Indeed, the prism hypotenuse is no more than $2.7 \mu \mathrm{m}$ and most of the incident light could pass around the prism to continue straight forward (Mie effect). To reduce this problem, we use a Gaussian shaped incident beam, with a full width at half maximum similar to the prism size. This corresponds to $1.8 \mu \mathrm{m}$. Using a narrower beam would confine the light still more inside the cavity, avoiding almost totally the lateral escape of energy. However, we choose this width to enlighten a reasonable surface portion and be able to compare our results with experimental observations. Moreover, the beam width cannot be smaller than the wavelength. The mirrors are both made of silver $[\epsilon=(-17.2,0.5)]$, which gives good reflectors with limited absorption. The semitransparent mirror is $50 \mathrm{~nm}$ thick, so that about $4 \%$ of the incident light can pass through it. The mirror is a little bit thicker: 60 $\mathrm{nm}$. Like this we ensure that the largest part of the light entering the resonator is trapped inside. To get the amplification coefficient $\alpha$, we compute the field at a definite point just below the surface. This is the place where the multiple interferences produce the maximal intensity inside the microresonator, as it will be presented in Fig. 5. This maximal intensity, normalized with the incident field intensity $\left|E_{0}\right|^{2}$, provides the amplification coefficient $\alpha$ reached inside the cavity. This coefficient $\alpha$ has a signification similar to the $Q$ factor of the cavity [19].

In our computation, we discretized the material domains with identical squares of $30-\mathrm{nm}$ sides. Such a discretization

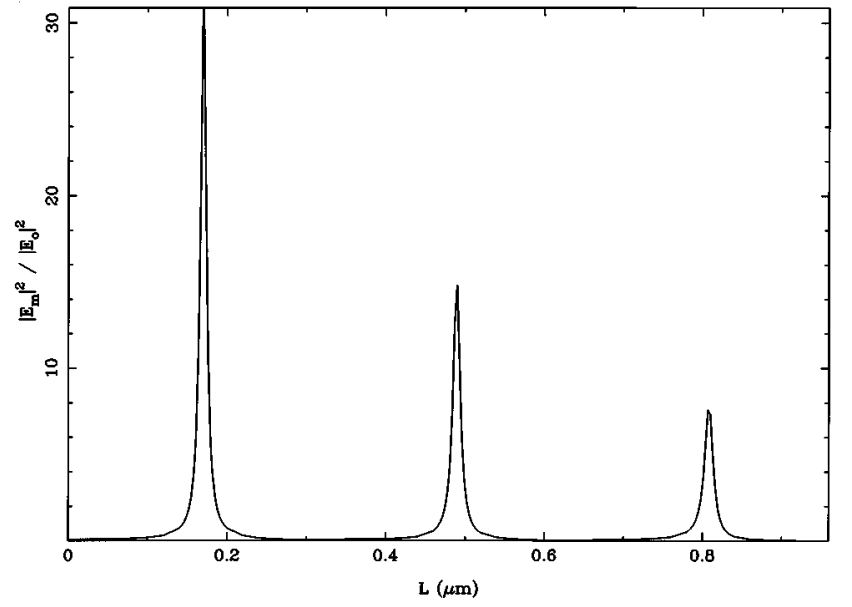

FIG. 3. Tuning curve of our microresonator as a function of the distance $L$ between the semitransparent mirror and the prism.

mesh verifies the convergence condition of our scalar algorithm: we must have around ten steps in each spatial direction per effective wavelength [10].

\section{Tuning the resonator}

As mentioned previously, the tuning of the resonator depends on the relative positions of the prism and the two mirrors. We choose to fix the position of the prism and the thick mirror as in Fig. 2 and we move the semitransparent mirror at a distance $L$ from the prism. For each position of the semitransparent mirror, we take the intensity of the electric field $15 \mathrm{~nm}$ below the prism surface, at a point where we know that the interference pattern presents a maximum for the current configuration.

The tuning curve obtained with this process is given in Fig. 3. Three successive resonances are presented. The first one is very sharp, with an amplification coefficient higher than 30 . The intensity of the following resonances decreases when the semitransparent mirror moves away from the prism. As a matter of fact, for a longer cavity, higher-order modes can be excited, and the larger the gap between the semitransparent mirror and the prism, the more light can escape laterally.

We will now devote our attention to the first resonance peak and consider three working points around this peak. The first point $\mathcal{A}$ will be the resonance point, when the semitransparent mirror is located at a distance of $170 \mathrm{~nm}$ from the prism. When moving slightly the semitransparent mirror away from the prism, the amplification coefficient falls down drastically. On this slope, we will consider two more working points. The point $\mathcal{B}$ corresponds to an amplification coefficient $\alpha$ equal to half the maximum amplification, whereas the point $\mathcal{C}$ corresponds to $\alpha=2$. For these three working points, we compute the surface standing wave $15 \mathrm{~nm}$ above the prism surface (Fig. 4). The periodicity of this surface wave remains the same for the three working points. Note that the periodicity of the standing wave just above the prism does not correspond to the periodicity of the resonant peaks in Fig. 3. This difference emphasizes the complexity of the system. Once more, effects related to the limited prism size appear on Fig. 4. Indeed, when approaching the corners of 


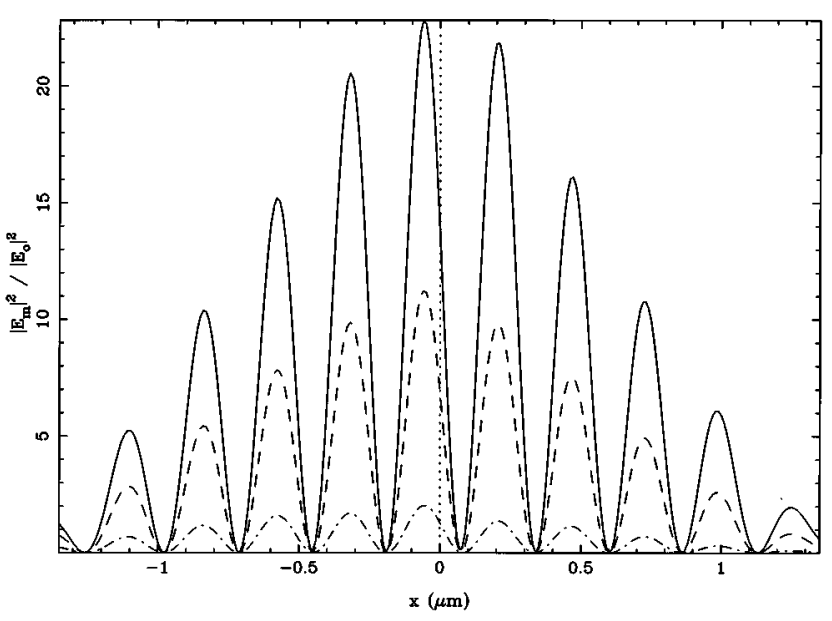

FIG. 4. Standing wave $15 \mathrm{~nm}$ above the prism surface for the three working points of the tuning curve. The solid curve is for $\mathcal{A}$ (resonance), the dashed curve for $\mathcal{B}$ (half-resonance), and the dashdotted curve for $\mathcal{C}$ (amplification $\alpha=2$ ).

the prism, the field intensity falls down.

It is interesting to note how this surface wave is related to the interferences occurring inside the prism. For this we give in Fig. 5 a map of the near-field amplitude $|\mathbf{E}(x, y)|$ inside and around the resonator. On this map, the semitransparent mirror is located at the first resonant position. This near-field pattern corresponds to the working point $\mathcal{A}$. The slight asymmetry of the interference pattern resulting from the multiple reflections is due to the asymmetry of the resonator. Highintensity spots are regularly spaced between the two mirrors. The maximal intensity, as given on the scale, shows an amplification factor of more than 30 and is located just under the prism surface, at the point considered previously for the computation of the amplification coefficient $\alpha$. One clearly sees in Fig. 5 that the surface standing wave is an extension to the outside of the interference pattern in the prism, just below the surface. Above the surface, the intensity decays exponentially. This decay can also be computed for our three working points.

In order to investigate this decay, we compute for the three considered working points the field intensity above the brightest spot, as a function of the distance to the surface

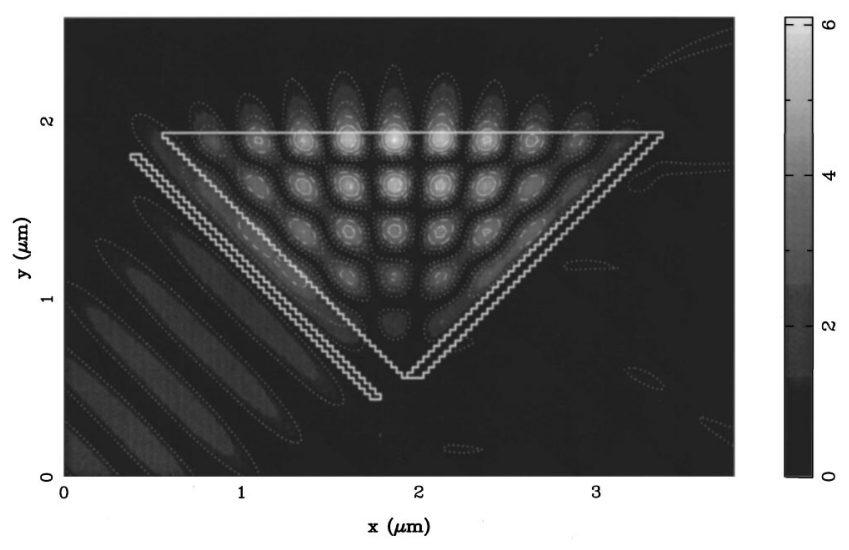

FIG. 5. Distribution of the near-field amplitude inside and around the microresonator when it is tuned.

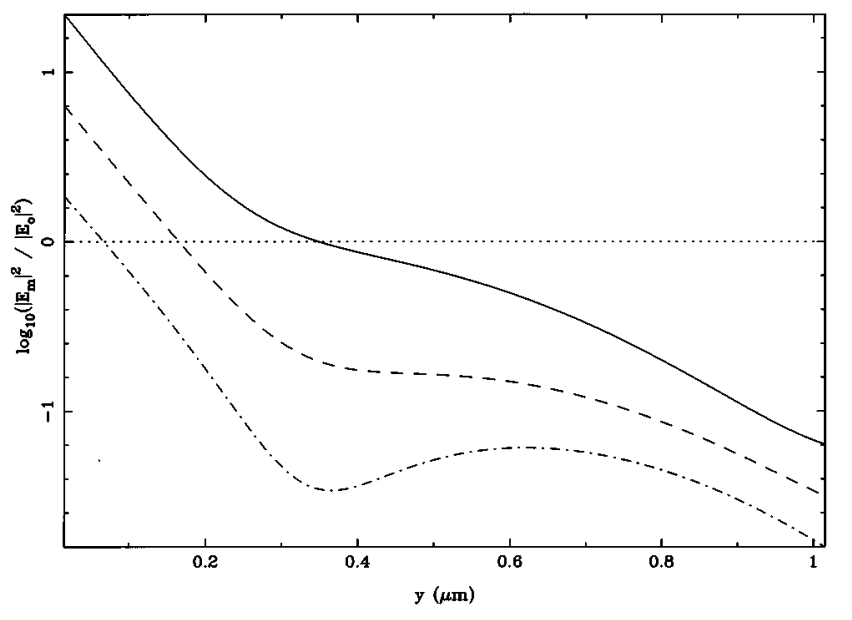

FIG. 6. Intensity decay above the resonator as a function of the distance $y$ from the resonator surface for the three working points $\mathcal{A}$ (solid curve), $\mathcal{B}$ (dashed curve), and $\mathcal{C}$ (dash-dotted curve).

(Fig. 6). A semilogarithmic representation of these curves presents two distinct parts for the $y$ dependence of the intensity. From the surface (located at $y=0$ ) up to $200 \mathrm{~nm}$, the dependence is, as expected, purely exponential. Above 200 $\mathrm{nm}$, the curve is modified because, once more, of a size effect. Indeed, part of the incident light passing around the system is added to the evanescent wave and more intensity than predicted by a pure total internal reflection process is observed at these larger distances. This size effect is more important for the nonresonant positions of the semitransparent mirror since more light passes around the resonator when it is detuned.

\section{Approaching a tip}

The fourth element of our multidomain system, a thin glass "pencil" tip $(\epsilon=2.25)$, is now placed at the vicinity of the microresonator. This kind of tip is similar to the chemically etched probes used in near-field optical microscopy [20]. Intentionally, we chose a reduced size probe so that it does not perturb the system too much and we did not cover its faces with a metallic coating. Our aim here is to demonstrate that even such a little perturbation added to our resonant system can strongly modify the characteristics of the system.

The interaction of a tip with a surface enlightened in total internal reflection was already studied by other methods $[21,22]$. The effect of the tip presence was expressed as a transfer function between the signal existing without a tip and the signal collected by the tip. Here we want to show how the perturbation induced by the tip modifies the nearfield pattern and, in particular, the resonant properties of our microcavity. In this way, we can visualize the coupling occurring between a tip and a surface, even if this tip is extremely sharp and often supposed as being passive.

Positioning the tip above the central bright spot of the surface (i.e., the spot corresponding to the highest intensity inside the microresonator), we progressively decrease the tipsurface distance. The influence of the probe increases as the distance to the microresonator decreases. The dependence of the microresonator amplification factor $\alpha$ on the tip-surface 


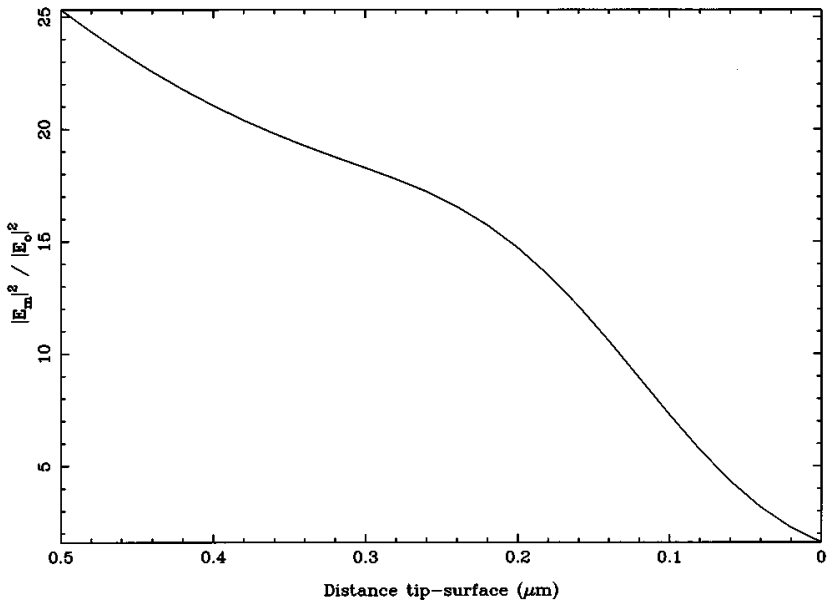

FIG. 7. Decrease of the maximal intensity inside the microresonator when a small external dielectric probe is approached to the prism surface.

distance is given in Fig. 7. The amplification coefficient is obtained here also by computing the maximum electric-field intensity in the prism. As we see in Fig. 7, the presence of the external tip is already felt inside the resonator at a relatively large tip-surface separation distance, leading to a decrease of the amplification factor $\alpha$. The smaller the separation distance, the more important this decrease of $\alpha$. At a separation distance equal to half the wavelength, we observe an inflection of the curve in Fig. 7. This inflection is related to an additional microcavity effect between the tip and the prism surface. This effect is maximum when the separation is on the order of half the wavelength. At very short separation distances, the presence of the tip breaks down the resonance of the system because the coupling with the entire tip becomes stronger.

Three different positions of the probe above the prism surface will now be considered, each of them corresponding to a different regime of the preceding approach curve. For each position, we will present the tuning curve of the coupled tip-microresonator system. For the closest position, we will also present the near-field pattern occurring inside this system when it is resonant. The tuning curves are here also obtained by moving the semitransparent mirror away from the prism and considering, for each position, the electric-field intensity at a definite point inside the microresonator where we know that the highest intensity is reached.

\section{E. Detuning the cavity}

We limit our calculations of the tuning curve to the first resonance peak. On Fig. 8, four tuning curves are presented, showing the progressive destruction of the resonance when the tip is approached. The reference curve, drawn in continuous line, corresponds to the first peak of Fig. 3, i.e., the tuning curve when the tip is located infinitely far away from the microresonator. The dashed curve, corresponding to a tip located $360 \mathrm{~nm}$ above the surface, is still very similar to the reference curve. The maximal amplification is slightly reduced, but the amplification factor $\alpha$ remains high. When the tip is approached at $150 \mathrm{~nm}$ from the surface (dash-dotted

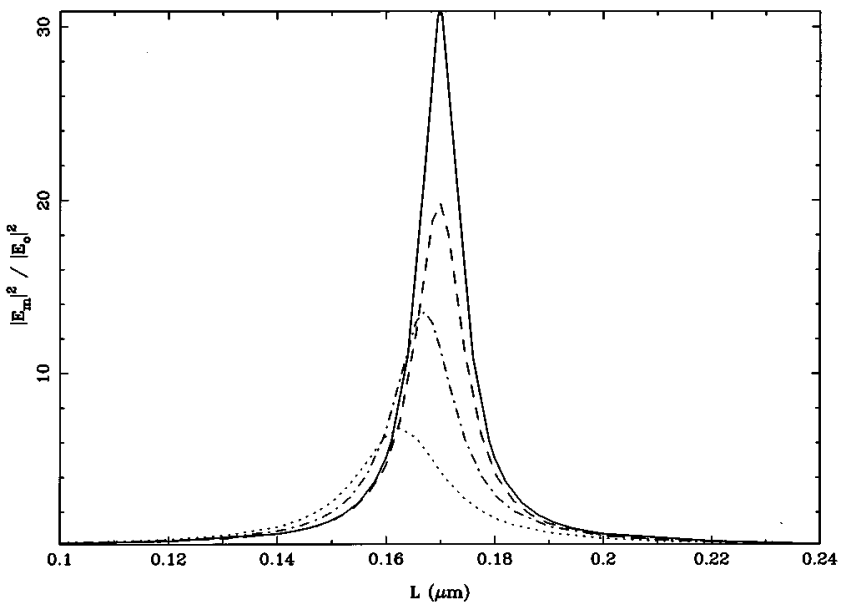

FIG. 8. Tuning curves obtained for the coupled tip-microcavity system. Three tip heights are considered: $360 \mathrm{~nm}$ (dashed curve), $150 \mathrm{~nm}$ (dash-dotted curve), and $60 \mathrm{~nm}$ (dotted curve). The tuning curve obtained for the microcavity alone is given for comparison (solid curve).

curve), we can no longer qualify our system as a "good resonator": the amplification factor $\alpha$ is strongly reduced by the coupling between the tip and the microresonator. The dotted curve, obtained for a tip-surface distance of $60 \mathrm{~nm}$, presents an even worse resonance. However, it is interesting to note that some weak exaltation remains, even when the tip is placed in the very near-field zone.

A second effect due to the tip is a slight shift of the resonant position to a smaller semitransparent mirror-prism distance. This shift is accentuated when the tip approaches the surface. It is the sign that a new resonator, resulting from the tip-microcavity coupling, is progressively created. This new cavity presents a resonance at a different relative position of its components. The shift of the resonance is partly hidden by the simultaneous intensity loss and broadening of the peak. It would be more appreciable if the amplification factor $\alpha$ of the new cavity was comparable to the one obtained without a tip.

Because we chose a small probe, the near-field pattern obtained inside the coupled tip-microresonator system (Fig. 9) is comparable to the one given in Fig. 5, when no tip was present. However, as proved by the scale, the maximum amplitude is reduced by a factor 2.5 , which is about a factor 6 in intensity. We also see that a small amount of light is transferred to the tip. This transfer is not so important because the tip is very thin and remains far from the surface $(60 \mathrm{~nm})$. Therefore the penetration of light inside the tip is not easy. A third noticeable effect is the relative increase of the reflection on the semitransparent mirror. This effect is simply due to the reduced field intensity in the prism, so that the standing wave occurring from the reflection at the semitransparent mirror appears to have a higher amplitude in Fig. 9, as it has in Fig. 5.

We repeated this calculation using tips that ended with smaller and smaller angles. To obtain a coupled cavity with comparable quality factor, we had to consider an aperture angle of $15^{\circ}$. When the tip is so sharp, the detuning is almost only marked by a shift of the resonance, the height and width of the resonance peak remaining important. A new cavity of 


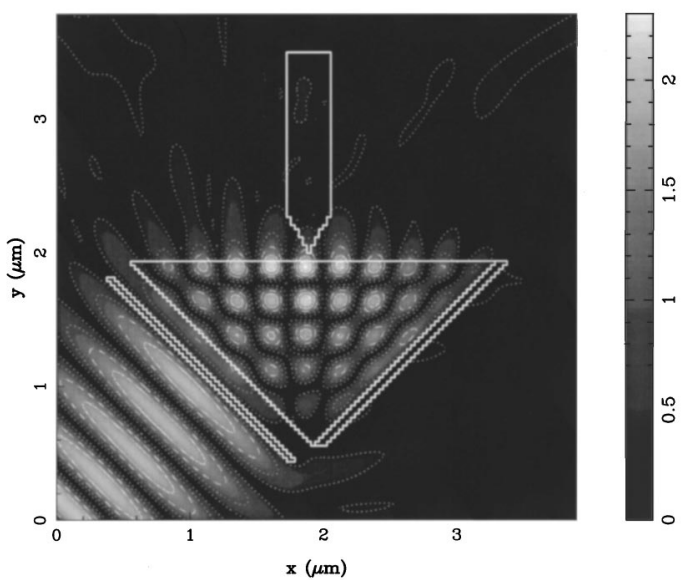

FIG. 9. Map of the electric-field amplitudes inside the coupled tip-microresonator system when the tip is $60 \mathrm{~nm}$ above the prism surface.

good quality is created by coupling this sharp tip with the prism. Let us remark that, even with this sharp tip, the quality of the resonator is still slightly reduced. The first resonance peak obtained for this coupled cavity, when the sharp tip if $60 \mathrm{~nm}$ away from the surface, is compared with the resonance peak of the microcavity alone in Fig. 10.

\section{F. Relation with recent experimental works}

The scheme of our microresonator was borrowed from an experiment realized two years ago by different teams working in the near-field microscopy world $[16,15]$. These people showed how the photon scanning tunneling microscope (PSTM) could be used to look inside a Fabry-Pérot cavity. This combination of near-field microscopy and interferometry also opens the outlook on a better resolution for the PSTM. It is possible to build an experimental setup consisting of a prism and two mirrors, as in Fig. 5, and presenting a quite narrow resonance for a precise relative position of the three elements. If we put these elements in a position very

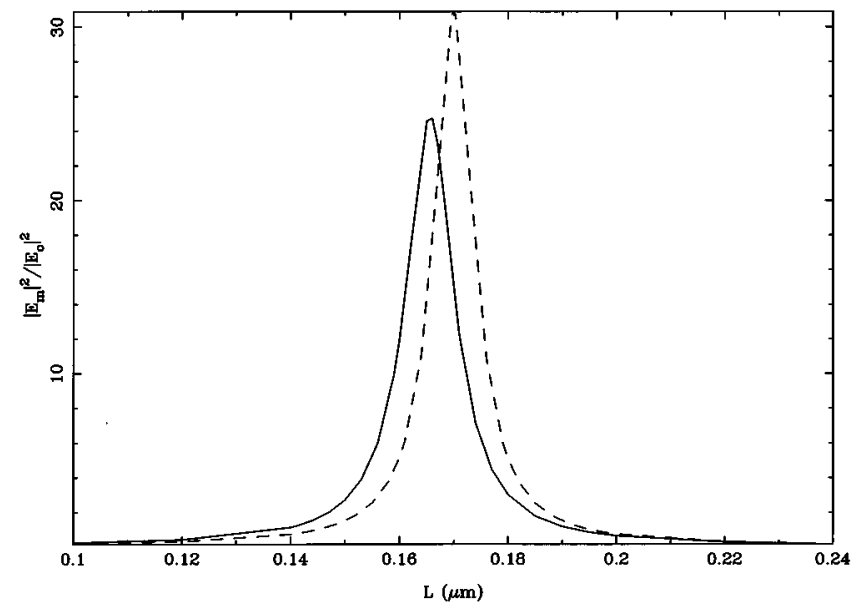

FIG. 10. Tuning curve obtained for a microcavity coupled with a very sharp tip (opening angle of $15^{\circ}$ ) located $60 \mathrm{~nm}$ above the prism surface. This tuning curve is compared with the one obtained for the microcavity alone (dashed curve). close to the resonant one, the system will be very unstable. Approaching a tip, as we showed before, will induce a coupling between the resonator and the tip and the creation of a new cavity. The high intensity of the surface evanescent wave will suddenly fall down because the resonant position is shifted. The whole system will be immersed in a dark field. In such conditions, the contribution of a sample deposited on a surface would be emphazised and one could hope to improve the resolution of the PSTM [24].

\section{CONCLUSION}

In this paper we presented a theoretical method particularly suited to study the interaction of light with any material system having mesoscopic dimensions. The method, based on a propagator technique, was applied to a general problem involving several dielectric domains mutually independent in the absence of any excitation. When applying an electromagnetic field on this material system, we distinguished the interactions established between different domains and the interactions occurring between the constitutive elements of a given domain.

In this $a b$ initio method, the only approximation lies in the discretization of each material domain. The reference medium was not discretized, so it was possible to move the different objects composing the system in a continuous way. This property was of importance in the application that we presented. We determined in this application the resonance of a mesoscopic Fabry-Pérot cavity. We considered also the perturbation induced in this resonance by the presence of a dielectric probe also having mesoscopic dimensions. These simulations were possible because we were able to impose on the involved objects extremely precise relative moves.

This theoretical work finds similarities in recent experiments using the new optical near-field techniques. The resonant modes of small cavities, the interferential behavior of waves in resonators are now accessible phenomena. We intend to go on with these modelizations and collaborate with experimentalists in order to get a better understanding of these fascinating near-field phenomena.

\section{ACKNOWLEDGMENTS}

The authors particularly appreciated helping discussions with Daniel Courjon, Michel Spajer, and Jean-Marie Vigoureux. O.J.F.M. gratefully acknowledges the support of the Swiss National Science Foundation (Grant No. PNR 36) and A.C. is grateful to the Belgian National Foundation for Research for financial support.

\section{APPENDIX A: FIELD SUSCEPTIBILITY FOR A 2D SYSTEM}

If the considered system has a translational invariance in the $z$ direction, its susceptibility does not depend any more on $z$ and can be written for each subdomain as

$$
\boldsymbol{\chi}_{n}(\mathbf{r}, \omega)=\boldsymbol{\chi}_{n}(\boldsymbol{\rho}, \omega),
$$

where $\boldsymbol{\rho}=(x, y)$. Therefore, it is possible to limit the entire calculation to the $(x, y)$ plane. Nonetheless, the perturbating optical field remains general: its incidence direction is not 
submitted to the space symmetry and any incident propagation vector $\mathbf{k}_{0}$ can be considered.

Let us use this symmetry to derive from the general 3D field susceptibility given in Eq. (10) a field susceptibility adapted to 2D problems. Starting from Lippmann-Schwinger equation (12), we apply a Fourier transform along the symmetry direction $z$. For each Fourier component $k_{z}$ we obtain the Lippmann-Schwinger equation

$$
\begin{aligned}
\mathbf{E}\left(\boldsymbol{\rho}, k_{z}, \omega\right) & \\
= & \mathbf{E}_{0}\left(\boldsymbol{\rho}, k_{z}, \omega\right) \\
& +\sum_{n=1}^{N} \int d \boldsymbol{\rho}^{\prime} \mathbf{S}_{0}\left(\boldsymbol{\rho}, \boldsymbol{\rho}^{\prime}, k_{z}, \omega\right) \cdot \boldsymbol{\chi}_{n}\left(\boldsymbol{\rho}^{\prime}, \omega\right) \cdot \mathbf{E}\left(\boldsymbol{\rho}^{\prime}, \omega\right) .
\end{aligned}
$$

The two-dimensional field susceptibility $\mathbf{S}_{0}\left(\boldsymbol{\rho}, \boldsymbol{\rho}^{\prime}, k_{z}, \omega\right)$ in Eq. (A2) is deduced from Eq. (10) by an inverse Fourier transform

$$
\mathbf{S}_{0}\left(\boldsymbol{\rho}, \boldsymbol{\rho}^{\prime}, k_{z}, \omega\right)=\int d\left(z-z^{\prime}\right) \mathbf{S}_{0}\left(\mathbf{r}, \mathbf{r}^{\prime}, \omega\right) e^{-i k_{z}\left(z-z^{\prime}\right)},
$$

which gives

$$
\mathbf{S}_{0}\left(\boldsymbol{\rho}, \boldsymbol{\rho}^{\prime}, k_{z}, \omega\right)=-\frac{\omega^{2}}{c^{2}} \int d \mathbf{k}_{\|}\left[\mathbf{1}-\frac{1}{k_{0}^{2}} \mathbf{k k}\right] \frac{e^{i \mathbf{k}_{\|} \cdot \mathbf{R}_{\|}}}{Q^{2}-k_{\|}^{2}} .
$$

The vectors $\mathbf{R}_{\|}$and $\mathbf{k}_{\|}$are, respectively, the projection of $\mathbf{R}$ and $\mathbf{k}$ on the $(x, y)$ plane and $Q^{2}=k_{0}^{2}-k_{z}^{2}$. The $-\omega^{2} / c^{2}$ factor is the constant connecting the field susceptibility to the Green's dyadic. Equation (A4) can be solved using polar coordinates. Indeed, if we assume

$$
\begin{array}{r}
\mathbf{k}_{\|}=\left(k_{\|} \cos \theta, k_{\|} \sin \theta\right), \\
\mathbf{R}_{\|}=\left(R_{\|} \cos \delta, R_{\|} \sin \delta\right)
\end{array}
$$

and develop the imaginary exponential in (A4) into a Bessel functions series

$$
\begin{aligned}
\exp \left[i k_{\|} R_{\|} \cos (\theta-\delta)\right]= & J_{0}\left(k_{\|} R_{\|}\right) \\
& +2 \sum_{m=1}^{\infty} i^{m} J_{m}\left(k_{\|} R_{\|}\right) \cos [m(\theta-\delta)],
\end{aligned}
$$

the angular part of the integral (A4) is directly solvable and the integral over the $k_{\|}$space can be obtained by the residue theorem. Finally, we obtain the form of the field susceptibility for a $2 \mathrm{D}$ system

$$
\mathbf{S}_{0}\left(\boldsymbol{\rho}, \boldsymbol{\rho}^{\prime}, k_{z}, \omega\right)=\frac{i}{4} \frac{\omega^{2}}{c^{2}}\left(\begin{array}{ccc}
S_{0}^{11} & S_{0}^{12} & S_{0}^{13} \\
S_{0}^{21} & S_{0}^{22} & S_{0}^{23} \\
S_{0}^{31} & S_{0}^{32} & S_{0}^{33}
\end{array}\right)
$$

with

$$
\begin{gathered}
S_{0}^{11}=\left[1-\frac{k_{0 \|}^{2}}{2 k_{0}^{2}}\right] H_{0}^{(1)}\left(k_{0 \|} R_{\|}\right)+\frac{k_{0 \|}^{2}}{2 k_{0}^{2}} \cos (2 \delta) H_{2}^{(1)}\left(k_{0 \|} R_{\|}\right), \\
S_{0}^{12}=\frac{k_{0 \|}^{2}}{2 k_{0}^{2}} \sin (2 \delta) H_{2}^{(1)}\left(k_{0 \|} R_{\|}\right)=S_{0}^{21}, \\
S_{0}^{13}=-\frac{i k_{0 \|} k_{0 z}}{k_{0}^{2}} \cos (\delta) H_{1}^{(1)}\left(k_{0 \|} R_{\|}\right)=S_{0}^{31}, \\
S_{0}^{22}=\left[1-\frac{k_{0 \|}^{2}}{2 k_{0}^{2}}\right] H_{0}^{(1)}\left(k_{0 \|} R_{\|}\right)-\frac{k_{0 \|}^{2}}{2 k_{0}^{2}} \cos (2 \delta) H_{2}^{(1)}\left(k_{0 \|} R_{\|}\right), \\
S_{0}^{23}=-\frac{i k_{0 \|} k_{0 z}}{k_{0}^{2}} \sin (\delta) H_{1}^{(1)}\left(k_{0 \|} R_{\|}\right)=S_{0}^{32}, \\
S_{0}^{33}=\left[1-\frac{k_{0 z}^{2}}{k_{0}^{2}}\right] H_{0}^{(1)}\left(k_{0 \|} R_{\|}\right) .
\end{gathered}
$$

The particular shape of this tensor when $R_{\|}$is zero is given in Appendix B.

\section{APPENDIX B: SINGULARITY OF THE FIELD SUSCEPTIBILITY}

When the source point, at $\mathbf{r}^{\prime}$, coincides with the target point, at $\mathbf{r}$, the expression of the field susceptibility, given in Eq. (A4), presents a singularity. We will show in this appendix how this singularity can be removed.

If we consider that the material system is a single cell centered in $\boldsymbol{\rho}_{i}$, the Lippmann-Schwinger equation (A2) can be written as

$$
\left[\mathbf{1}-\mathbf{S}_{0}\left(\boldsymbol{\rho}_{i}, \boldsymbol{\rho}_{i}, \omega\right) \boldsymbol{\chi}\left(\boldsymbol{\rho}_{i}, \omega\right) w_{i}\right] \mathbf{E}\left(\boldsymbol{\rho}_{i}, \omega\right)=\mathbf{E}_{0}\left(\boldsymbol{\rho}_{i}, \omega\right),
$$

where $w_{i}$ is the weight associated with the unit cell. In this two-dimensional case, this weight is the surface of the cell. Since the discretization cell has a finite dimension, it undergoes a depolarization effect. We have to include this effect when evaluating the field. The following equation, relating field and polarization, has to be verified:

$$
\mathbf{E}\left(\boldsymbol{\rho}_{i}, \omega\right)=\mathbf{E}_{0}\left(\boldsymbol{\rho}_{i}, \omega\right)-\mathbf{L}_{2 \mathrm{D}} \frac{\mathbf{P}\left(\boldsymbol{\rho}_{i}, \omega\right)}{\epsilon_{0}} .
$$

The corrective terms to remove from the propagator dyadic have been tabulated for different kinds of discretization [5]. This tensor is, for a material system being invariant following the $z$ axis and discretized by infinitely long square rods [23],

$$
\mathbf{L}_{2 \mathrm{D}}=\left(\begin{array}{ccc}
\frac{1}{2} & 0 & 0 \\
0 & \frac{1}{2} & 0 \\
0 & 0 & 0
\end{array}\right)
$$

The total field susceptibility $\mathbf{S}_{0}^{\prime}$ can be written, when adding this depolarization contribution to the dyadic $\mathbf{S}_{0}$ presented in Eq. (A8), as 


$$
\mathbf{S}_{0}^{\prime}\left(\boldsymbol{\rho}, \boldsymbol{\rho}^{\prime}, k_{z}, \omega\right)=\mathbf{S}_{0}\left(\boldsymbol{\rho}, \boldsymbol{\rho}^{\prime}, k_{z}, \omega\right)-\mathbf{L}_{2 \mathrm{D}} \delta\left(\boldsymbol{\rho}-\boldsymbol{\rho}^{\prime}\right) .
$$

The first part of the left member $\mathbf{S}_{0}$ seems to remain singular when $\boldsymbol{\rho}=\boldsymbol{\rho}^{\prime}$. This would be true if the considered source and target were pointlike. However, due to the discretization, each source point is extended over a discretization mesh. In order to evaluate the field susceptibility for the self-term, we have to integrate Eq. (A8) on that unit cell. In our twodimensional case, the integration zone is a square. We approximate it by a circle of radius $a$. When we integrate every component of the field susceptibility dyadic, we obtain zero, except for diagonal terms. The integration of these components gives

$$
S_{\mathrm{int}}=\frac{i}{4} \frac{\omega^{2}}{c^{2}}\left[1-\frac{\alpha}{2 k_{0}^{2}}\right]\left[a k_{0 \|} H_{1}^{(1)}\left(a k_{0 \|}\right)+\frac{2 i}{\pi}\right] \frac{2 \pi}{k_{0 \|}},
$$

where $\alpha$ is $k_{0 \|}^{2}$ for $S_{0}^{11}$ and $S_{0}^{22}$, and $2 k_{0 z}^{2}$ for $S_{0}^{33}$, and where $H_{1}^{1}$ is the Hankel function of first order and first kind. To conserve the dimensions during the discretized procedure, we have to normalize this term, as well as the depolarization tensor, by the cell weight $w_{i}$. The dyadic replacing $\mathbf{S}_{0}$ when the source and target coincide takes the final form

$$
\mathbf{S}_{0}\left(\boldsymbol{\rho}, \boldsymbol{\rho}, k_{z}, \omega\right)=\frac{1}{w_{i}}\left(\begin{array}{ccc}
S_{\mathrm{int}}-\frac{1}{2} & 0 & 0 \\
0 & S_{\mathrm{int}}-\frac{1}{2} & 0 \\
0 & 0 & S_{\mathrm{int}}
\end{array}\right)
$$

When the dimension of the discretization cell tends to zero, the integrated part $S_{\text {int }}$ falls to zero, but the depolarization term remains.
[1] F. Pincemin, A. Sentenac, and J.-J. Greffet, J. Opt. Soc. Am. A 11, 1117 (1993).

[2] R. Carminati, A. Madrazo, and M. Nieto-Vesperinas, Opt. Commun. 111, 26 (1994).

[3] Ch. Girard and A. Dereux, Phys. Rev. B 49, 11344 (1994).

[4] R. E. Collin, Field Theory of Guided Waves, 2nd ed. (IEEE, New York, 1991).

[5] A. D. Yaghjian, Proc. IEEE 68, 248 (1980).

[6] G. S. Agarwal, Phys. Rev. A 11, 230 (1975).

[7] A. D. Maclachlan, R. D. Gregory, and M. A. Ball, Mol. Phys. 7, 119 (1963).

[8] Ch. Girard, Pure Appl. Opt. 1, 157 (1992).

[9] P. Morse and H. Feshbach, Methods of Theoretical Physics (McGraw-Hill, New York, 1953).

[10] O. J. F. Martin, A. Dereux, and Ch. Girard, J. Opt. Soc. Am. A 11, 1073 (1994).

[11] O. J. F. Martin, Ch. Girard, and A. Dereux, Phys. Rev. Lett. 74, 526 (1995).

[12] D. W. Pohl, Adv. Opt. Electron Microsc. 12, 244 (1991).

[13] F. de Fornel, L. Salomon, P. Adam, E. Bourillot, J. P. Goudonnet, and M. Nevière, Ultramicroscopy 42-44, 422 (1992).
[14] N. F. Van Hulst, M. H. P. Moers, and B. Bögler, J. Microscopy 171, 95 (1993).

[15] D. Courjon, C. Bainier, and F. Baida, Opt. Commun. 110, 7 (1994).

[16] A. J. Meixner, M. A. Bopp, and G. Tarrach, Appl. Opt. 33, 7995 (1994).

[17] A. Castiaux, Ch. Girard, A. Dereux, X. Bouju, and J. P. Vigneron, in Optics at the Nanometer Scale, Vol. 319 of NATO Advanced Study Institute, Series E, edited by M. Nieta Vesperinas and N. García (Kluwer, Dordrecht, 1996).

[18] N. Dubreuil, J. C. Knight, D. K. Leventhal, V. Sandoghdar, J. Hare, and V. Lefèvre, Opt. Lett. 20, 813 (1995).

[19] A. E. Siegman, Lasers (University Science Books, Mill Valley, CA, 1986).

[20] M. Spajer and A. Jalocha, in Near-Field Optics, Vol. 242 of NATO Advanced Studies Institute, Series E, edited by D. W. Pohl and D. Courjon (Kluwer, Dordrecht, 1993).

[21] R. Carminati and J.-J. Greffet, Opt. Commun. 116, 316 (1995).

[22] A. Madrazo and M. Nieto-Vesperinas, J. Opt. Soc. Am. A 13, 785 (1996).

[23] C. Kittel, Introduction to Solid State Physics (Wiley, New York, 1976).

[24] D. Courjon (private communication). 